\title{
Brugada Phenocopy in methanol toxicity: A novel marker of mortality
}

\author{
Mohammad Hossein Nikoo ${ }^{1}$, Alireza Estedal ${ }^{1}$, Kiana Khatami ${ }^{1}$, Maryam Pakfetrat ${ }^{1}$, \\ Alireza Arjangzadeh ${ }^{1}$, Shahrokh Sadeghi Boogar ${ }^{1}$, Sina Danesh ${ }^{1}$, and Seyyed Taghi \\ Heydari $^{1}$ \\ ${ }^{1}$ Shiraz University of Medical Sciences
}

October 10, 2020

\begin{abstract}
Introduction: Brugada phenocopies are recently introduced as clinical entities with electrocardiographic patterns identical to true congenital Brugada syndrome in individuals, who are asymptomatic and have no other clinical criteria. One of the largest methanol poisoning outbreaks occurred in Iran during March and April 2020. This study aimed to investigate the relationship between Brugada Phenocopy with laboratory data and demographic factors and determine its association with mortality rate in methanol toxicity patients. Method and results: Demographic data such as age, gender, medical history, Glasgow coma scale score, laboratory data, and electrocardiographic data were obtained from 356 patients, who were diagnosed with methanol poisoning and were hospitalized in Faghihi and Namazi Hospitals in Shiraz, southern Iran, during March and April 2020. The follow-up data was gathered to examine the development of any complications or death. A total of $20(5.6 \%)$ patients were admitted with a diagnosis of methanol toxicity and concurrent Brugada-like ECG patterns. Mortality rate, Glasgow Coma Scale Score $<3$, and blood sugar were significantly higher in the Brugada phenocopies; however, PH, O2 saturation, and calcium were lower among Brugada phenocopies in comparison to methanol toxicity patients with no Brugada pattern $(\mathrm{P}<0.05)$. Conclusion: According to the findings of this study, some recognizable ECG patterns and laboratory data may be used as novel prognostic factors of morbidity and mortality. Electrocardiography machines are widely available, and electrocardiograms can easily be obtained and interpreted. This may provide the grounds for caregivers to predict and prevent the incoming death and react more properly and timely.
\end{abstract}

\section{Hosted file}

Brugada-final.pdf available at https://authorea.com/users/366186/articles/485982-brugadaphenocopy-in-methanol-toxicity-a-novel-marker-of-mortality

\section{Hosted file}

Brugada tables.pdf available at https://authorea.com/users/366186/articles/485982-brugadaphenocopy-in-methanol-toxicity-a-novel-marker-of-mortality 